\title{
Targeting the aryl hydrocarbon receptor/polyamine biosynthesis axis of evil for cancer therapy
}

\author{
Robert A. Casero Jr.
}

Department of Oncology, Johns Hopkins University School of Medicine, and the Sidney Kimmel Comprehensive Cancer Center at Johns Hopkins, Baltimore, Maryland, USA. bind to AZ, block its inactivation/degradation of ODC, and increase the polyamine biosynthetic capacity of the cell.

Both ODC and AZIN1 have been implicated as oncogenes (8-10). Consequently, pathways leading to increased expression of these two proteins have the potential to lead to loss of growth control and carcinogenesis.

\section{Identifying AHR as a transcriptional regulator of polyamine biosynthesis}

Bianchi-Smiraglia et al. identified a new signaling pathway that activates the transcription of both ODC1 and AZIN1. Using bioinformatics, they identified AHR binding sites in the promoters of both genes. They then demonstrated that AHR directly activates the transcription of both $O D C 1$ and AZIN1, with the ectopic expression of a constitutively active form of AHR and by ChIP analysis in WI-38 human fibroblasts. Luciferase constructs confirmed an active transcriptional role of AHR in both genes in response to a cotransfected, activated AHR construct or treatment with benzo[a]pyrene, a known activating ligand of AHR. Next, since ODC1 is a known transcriptional target of MYC and because MYC itself has been implicated as a target of AHR, they went on to show that mutating the MYC-binding element in the ODC1 promoter had no effect on AHR-mediated transcription. Finally, to confirm that AHR was directly involved in ODC1 and AZIN1 expression, they either used siRNA or a known AHR antagonist, CH223191, to reduce AHR levels. In both cases, the expression levels of ODC1 and AZIN1 decreased and were accompanied by a decrease in polyamines.

These results are provocative for multiple reasons: (a) They suggest that AHR interference could be an effective targeting strategy to reduce ODC1 and AZIN1 expression; (b) they indicate that if there are neoplasms that rely on this signaling pathway for the increase in polyamines 

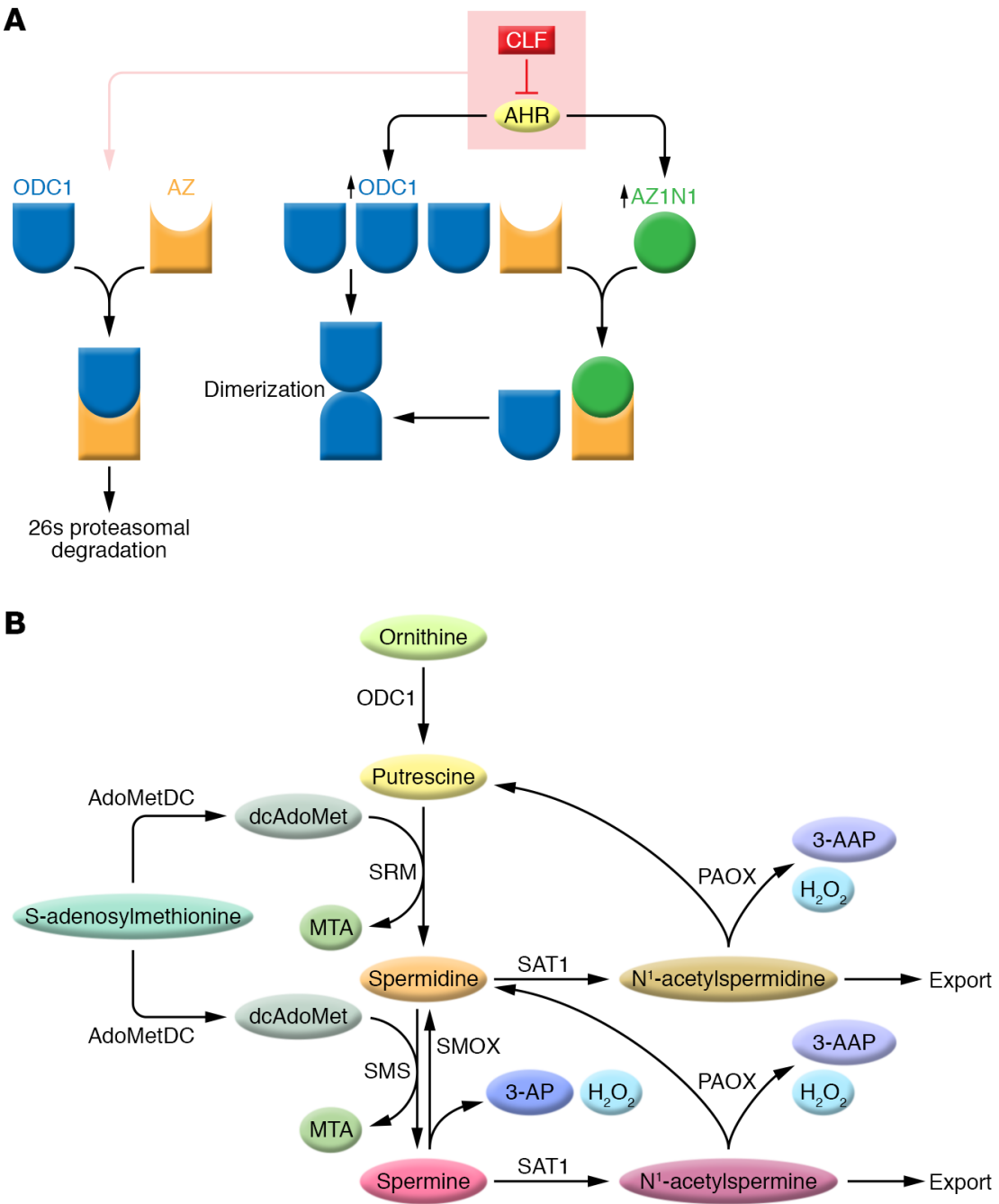

Figure 1. The regulatory dance of ODC1 in polyamine metabolism. (A) AHR increases the transcription of ODC1 and AZIN1, leading to increased ODC1 and AZIN1 protein levels (blue and green, respectively). This results in increased levels of the active ODC1 dimers and a reduction of AZ-inactivated ODC1 (orange). CLF blocks the transcriptional activation of AHR, thus favoring ODC1-AZ interactions leading to ODC degradation and reduced polyamines. (B) In this simplified polyamine metabolic pathway, ODC1 is a rate-limiting step in polyamine biosynthesis and produces the diamine 1,4-diaminobutane (putrescine). Spermidine synthase (SRM) and spermine synthase (SMS) transfer an aminopropyl group from decarboxylated S-adenosylmethionine (dcAdoMet) produced by the decarboxylation of S-adenosylmethionine for the synthesis of spermidine and spermine, respectively, with methylthioadenosine (MTA) as a byproduct of both reactions. SAT1 catalyzes the transfer of the acetyl group of acetyl coenzyme A to either spermidine or spermine, which are either excreted from the cell or serve as substrates for $\mathrm{N}^{1}$-acetylpolyamine oxidase (PAOX), a peroxisomal enzyme. 3-acetylaminopropanal (3-AAP) and $\mathrm{H}_{2} \mathrm{O}_{2}$ are byproducts of oxidation of the acetylated polyamines by PAOX. SMOX is a cytosolic and nuclear amine oxidase that directly oxidizes spermine to produce spermidine. 3-aminopropanal (3-AP) and $\mathrm{H}_{2} \mathrm{O}_{2}$ are byproducts of the oxidation of spermine by SMOX. Data presented by Bianchi-Smiraglia et al. indicate that CLF treatment of multiple myeloma cells decreases ODC1 activity and may increase both SAT1 and SMOX activity, thus reducing intracellular polyamines. For a more complete overview of polyamine metabolism, see ref. 1 . necessary for tumor proliferation, AHR signaling may be a potential target for anticancer therapy. Importantly, although AHR is postulated here to only affect the biosynthetic pathway, specifically, the production of the polyamine putrescine, the data indicate a rapid and significant depletion of all three polyamines when AHR is inhibited. If only polyamine biosynthesis were inhibited, the loss of the higher polyamines would only occur through dilution by division. However, the rapid polyamine depletion suggests that inhibiting AHR also has a role in inducing polyamine catabolism (Figure 1, and see below) (11).

\section{Identifying an effective drug targeting AHR to reduce polyamine biosynthesis}

Since their data are consistent with the hypothesis that AHR signaling is a rational drug target, Bianchi-Smiraglia et al. went on to identify small-molecule AHR antagonists useful in targeting polyamine metabolism. Only one drug, clofazimine (CLF), demonstrated a dose-dependent decrease in ODC1 and AZIN1 within 2 hours of treatment in WI-38 fibroblasts, similar to the effects of $\mathrm{CH} 223191$. Since CLF is a clinically approved drug for the treatment of leprosy, the authors extended their studies to confirm the mechanism of action of CLF and its potential utility as an antineoplastic agent.

Importantly, CLF treatment decreased all polyamines, again suggesting inhibition of polyamine biosynthesis and induction of the polyamine catabolic pathway. Thus, in addition to showing that ODC expression was down, the authors also provide evidence that the two important enzymes in polyamine catabolism, spermidine/ spermine $\mathrm{N}^{1}$-acetyltransferase (SAT1) and spermine oxidase (SMOX) are increased, thus explaining the rapid decrease in polyamines. Interestingly, SMOX has multiple putative AHR binding sites in its promoter region, suggesting that its transcription may also be regulated by AHR. However, in the case of SMOX, AHR would have to be a transcriptional repressor to respond to CLF with increased expression (https://www. encodeproject.org//).

The role of AHR in cellular pathology is complicated; it has been described as both a tumor suppressor and an oncogene $(12,13)$. To determine the subset of cancers that might benefit from targeting AHR, the authors surveyed 26 different cancer data sets, identified three specific tumor types whose expression of AHR was inversely correlated with survival, and chose multiple myeloma as their model. To confirm the link between AHR expression and polyamine metabolism in myeloma patients, they devised a polyamine biosynthesis score based on gene expression data from a patient data set. Patients with the highest expression of AHR and high- 
est polyamine biosynthesis score had the poorest survival.

To further confirm the link between AHR, polyamines, and myeloma, three different human myeloma cell lines, MM.1S, RPMI-8226, and U266, were used to recapitulate their findings in the WI-38 cells. Significantly, since U266 does not express AHR, it served as a negative control. While pharmacologic or shRNA-mediated inhibition of AHR signaling was effective in reducing cell growth and polyamine biosynthesis in the MM.1S and RPMI- 8226 cell lines, it had little effect in U266 cells. Further, addition of spermidine to CLF-treated cells partially rescued them from the growth-inhibitory effects of the AHR antagonist. There also was no evidence that MYC played a role in the response to interference with AHR signaling.

\section{CLF is effective in treating bortezomib-resistant multiple myelomas}

Bortezomib (BTZ) is a front-line therapy for multiple myeloma; however, relapse with BTZ resistance occurs frequently (14). To determine whether BTZ-resistant cell lines were susceptible to CLF, the authors generated BTZ-resistant clones of MM.1S and RPMI-8226. The results of these studies indicate that BTZ-resistant myeloma cells maintain their sensitivity to CLF. Unfortunately, combination studies of BTZ-resistant and -sensitive cells were not reported here.

Finally, CLF activity was evaluated in xenograft mouse models of MM.1S and RPMI-8226. The in vivo results confirmed the efficacy of CLF in the treatment of these models and demonstrated similar efficacy for BTZ. Additional confirmation of CLF activity was demonstrated in a $\mathrm{Vk}^{*} \mathrm{MYC}$ mouse model that spontaneously develops multiple myeloma. Here, too, CLF significantly reduced disease burden with a response comparable to that elicited by BTZ.

\section{Conclusions}

The results of these studies are very encouraging in that they identify an entirely new target and drug for malignancies such as multiple myeloma. The high expression of AHR and the dependence on polyamines for growth and survival in myeloma suggest a promising way forward in treating difficult diseases that express high levels of AHR. What would also be very interesting to know is the effect of drug combinations on these tumor models. The combination of BTZ and CLF should certainly be considered. Even more important would be the combination of CLF and DFMO. DFMO is clinically approved for the treatment of infection with a specific species of trypanosome, the parasite responsible for African sleeping sickness (15). DFMO is extremely well tolerated and is actively being studied in clinical trials as a chemopreventive agent in gastrointestinal and other cancers (16, 17) and in combination with other agents in the treatment of neuroblastoma and other malignancies (18-20). The idea of targeting ODC expression and activity at multiple steps, from transcription to stability to activity, is attractive, because the development of resistance to all mechanisms occurring at once is unlikely. Finally, it would be interesting to determine whether induction of polyamine catabolism is essential for the antitumor effect of CLF, as has been indicated for several antitumor polyamine analogs (21).

\section{Acknowledgments}

The author thanks Tracy Murray Stewart (Johns Hopkins University School of Medicine) for her help in preparing this Commentary. Work in the Casero laboratory is supported by grants from the $\mathrm{NIH}$ (CA204345), the Maryland Cigarette Restitution Fund, and the Samuel Waxman Cancer Research Foundation.

Address correspondence to: Robert A. Casero Jr., Department of Oncology, Johns Hopkins University School of Medicine and the Sidney Kimmel Comprehensive Cancer Center at Johns Hopkins, 1650 Orleans Street, Room 551, Baltimore, Maryland 21231, USA. Phone: 410.955.8580; Email: rcasero@jhmi.edu.

1. Casero RA, Murray-Stewart T, Pegg AE. Polyamines metabolism and cancer: treatments achallenges and opportunities [published online ahead of print September 4, 2018]. Nat Rev Cancer. https://doi.org/10.1038/s41568-018-0050-3.

2. Horn Y, Schechter PJ, Marton LJ. Phase I-II clinical trial with alpha-difluoromethylornithine - an inhibitor of polyamine biosynthesis. Eur J Cancer Clin Oncol. 1987;23(8):1103-1107.

3. Bianchi-Smiraglia A, et al. Inhibition of the aryl hydrocarbon receptor/polyamine biosynthesis axis suppresses multiple myeloma. J Clin Invest. 2018;128(10):4682-4696.

4. Kahana C. Antizyme and antizyme inhibitor, a regulatory tango. Cell Mol Life Sci. 2009;66(15):2479-2488.

5. Pegg AE. Regulation of ornithine decarboxylase. J Biol Chem. 2006;281(21):14529-14532.

6. Kahana C. Regulation of cellular polyamine levels and cellular proliferation by antizyme and antizyme inhibitor. Essays Biochem. 2009;46:47-61.

7. Kahana C. Protein degradation, the main hub in the regulation of cellular polyamines. Biochem J. 2016;473(24):4551-4558.

8. Gilmour SK. Polyamines and nonmelanoma skin cancer. Toxicol Appl Pharmacol. 2007;224(3):249-256.

9. Qiu S, Liu J, Xing F. Antizyme inhibitor 1: a potential carcinogenic molecule. Cancer Sci. 2017;108(2):163-169.

10. Shigeyasu K, et al. AZIN1 RNA editing confers cancer stemness and enhances oncogenic potential in colorectal cancer. JCI Insight. 2018;3(12):e99976.

11. Casero RA, Pegg AE. Polyamine catabolism and disease. Biochem J. 2009;421(3):323-338.

12. Formosa R, Borg J, Vassallo J. Aryl hydrocarbon receptor (AHR) is a potential tumour suppressor in pituitary adenomas. Endocr Relat Cancer. 2017;24(8):445-457.

13. Kolluri SK, Jin UH, Safe S. Role of the aryl hydrocarbon receptor in carcinogenesis and potential as an anti-cancer drug target. Arch Toxicol. 2017;91(7):2497-2513.

14. Wallington-Beddoe CT, Sobieraj-Teague M, Kuss BJ, Pitson SM. Resistance to proteasome inhibitors and other targeted therapies in myeloma. Br J Haematol. 2018;182(1):11-28.

15. Heby O, Persson L, Rentala M. Targeting the polyamine biosynthetic enzymes: a promising approach to therapy of African sleeping sickness, Chagas' disease, and leishmaniasis. Amino Acids. 2007;33(2):359-366.

16. Laukaitis CM, Erdman SH, Gerner EW. Chemoprevention in patients with genetic risk of colorectal cancers. Colorectal Cancer. 2012;1(3):225-240.

17. Meyskens FL, Simoneau AR, Gerner EW. Chemoprevention of prostate cancer with the polyamine synthesis inhibitor difluoromethylornithine. Recent Results Cancer Res. 2014;202:115-120.

18. Levin VA, Ictech SE, Hess KR. Clinical importance of eflornithine ( $\alpha$-difluoromethylornithine) for the treatment of malignant gliomas. CNS Oncol. 2018;7(2):CNS16.

19. Rounbehler RJ, Li W, Hall MA, Yang C, Fallahi M, Cleveland JL. Targeting ornithine decarboxylase impairs development of MYCN-amplified neuroblastoma. Cancer Res. 2009;69(2):547-553.

20. Saulnier Sholler GL, et al. A phase I trial of DFMO targeting polyamine addiction in patients with relapsed/refractory neuroblastoma. PLoS One. 2015;10(5):e0127246.

21. Casero RA Jr, Woster PM. Recent advances in the development of polyamine analogues as antitumor agents. JMed Chem. 2009;52(15):4551-4573. 